\title{
Comprehensive assessment of oncological outcomes in 186 patients with high-risk non-muscle-invasive bladder cancer: A single institution retrospective study
}

\author{
YUTO MATSUSHITA, KEITA TAMURA, DAISUKE MOTOYAMA, TOSHIKI ITO, \\ TAKAYUKI SUGIYAMA, ATSUSHI OTSUKA and HIDEAKI MIYAKE
}

Department of Urology, Hamamatsu University School of Medicine, Higashi-ku, Hamamatsu, Shizuoka 431-3192, Japan

Received June 1, 2019; Accepted June 1, 2020

DOI: $10.3892 / \mathrm{mco} .2020 .2086$

\begin{abstract}
The aims of the current study were to analyze the oncological outcomes of patients with high-risk non-invasive bladder cancer (NMIBC) and to identify prognostic factors in these patients. The present study included 186 consecutive patients who underwent transurethral resection of the bladder tumor (TURBT) between January 2007 and June 2017 at Hamamatsu University School of Medicine and were subsequently diagnosed with high-risk NMIBC according to the classification of the European Urological Association guidelines. The oncological outcomes, including recurrence-free survival (RFS), progression-free survival (PFS) and overall survival (OS) in the 186 patients were evaluated. Additionally, the effects of several clinicopathologiocal parameters on these outcomes were investigated. After the initial TURBT, the second transurethral resection and intravesical bacillus of Calmette-Guerin (BCG) therapy were performed for 47 (25.3\%) and $108(58.1 \%)$ patients, respectively. During the observation period of the current study, disease recurrence, disease progression and overall deaths occurred in 54 (29.0\%), $14(7.5 \%)$ and $19(10.2 \%)$ patients, respectively. The 5-year RFS, PFS and OS rates in the 186 patients were 66.6, 90.2 and $87.2 \%$, respectively. Multivariate analyses using the Cox proportional hazards regression model identified the following independent factors for the oncological outcomes: Tumor multiplicity and introduction of BCG therapy for RFS $(\mathrm{P}=0.018$ and $\mathrm{P}<0.008$, respectively), tumor multiplicity and recurrence status for $\mathrm{PFS}(\mathrm{P}=0.043$ and $\mathrm{P}=0.029$, respectively), and age and tumor multiplicity for $\mathrm{OS}(\mathrm{P}<0.008$ and $\mathrm{P}=0.041$, respectively). Although management following initial TURBT
\end{abstract}

Correspondence to: Dr Yuto Matsushita, Department of Urology, Hamamatsu University School of Medicine, 1-20-1 Handayama, Higashi-ku, Hamamatsu, Shizuoka 431-3192, Japan

E-mail: yuto.m@hama-med.ac.jp

Key words: high-risk non-muscle-invasive bladder cancer, recurrence-free survival, progression-free survival, overall survival, tumor multiplicity was insufficient, the oncological outcomes in the present series were comparable to those in previous studies targeting high-risk patients with NMIBC. However, attention should be paid to patients with factors independently associated with poor prognostic outcomes, particularly those with multiple tumors.

\section{Introduction}

At the initial diagnosis, approximately $75 \%$ of patients with urothelial carcinoma of the bladder have non-muscle invasive disease, which is generally treated by transurethral resection of the bladder tumor (TURBT) either alone or in combination with intravesical instillation therapy (1). However, it has been well documented that non-muscle invasive bladder cancer (NMIBC) is characterized by diverse biological and clinical features (2). Indeed, the prognosis of NMIBC patients is generally favorable, with survival rates $>80 \%$ at 5 years after the initial TURBT. However, recurrence rates of NMIBC patients range from 50-70 and 10-15\% of these patients develop muscle invasive disease (3-5). Accordingly, it is very important to perform detailed assessment of oncological outcomes of NMIBC patients with a high risk of disease recurrence and progression.

To date, multiple risk factors for NMIBC, including tumor size, number of tumors, grade, stage, prior recurrent status and concomitant carcinoma in situ (CIS), have been identified (6). Furthermore, several systems have been developed by incorporating such prognostic characteristics in order to facilitate risk prediction and guide therapeutic strategies for each NMIBC patient, such as the European Organization for Research and Treatment of Cancer (EORTC) and the Spanish Urological Club for Oncological Treatment (CUETO) scoring systems (6-8). However, although these systems have been proposed, it remains difficult to timely provide optimal treatment to NMIBC patients, particularly high-risk patients $(9,10)$. For example, Xylinas et al analyzed the significance of the EORTC and CUETO systems based on the data from 4689 patients with NMIBC, and found that these systems provide low positive predictive values for disease recurrence and progression, resulting in the overestimation of the progression risk in high-risk patients (10). 
Considering these findings, to precisely characterize the prognostic status in high-risk NMIBC patients treated in routine clinical practice, we retrospectively evaluated the oncological outcomes in a total of 186 consecutive Japanese patients with high-risk NMIBC who underwent TURBT at our institution, and investigated the prognostic impact of several clinicopathological parameters in these patients.

\section{Patients and methods}

Ethics approval. The design of the present study was approved by the research Ethics Committee of our institution (No. 14-290). The need to obtain informed consent from the included patients was waived because of its retrospective design; however, an opportunity for opt-out regarding this study was provided through the web site at our institution. In this study, a standard linkable de-identification of data was conducted by masking personal identifiers for privacy protection.

Patients. After excluding patients with an observation period $<1$ month after TURBT, this study included a total of 186 consecutive patients who underwent TURBT between January 2007 and June 2017 at our institution. They were pathologically diagnosed with NMIBC, and were classified as having high-risk disease based on the European Urological Association (EAU) guidelines (4). All clinicopathological data used in this study were obtained from the medical records for each patient. In this series, recurrence-free survival (RFS), progression-free survival (PFS) and overall survival (OS) were defined as the length of time from performed TURBT to pathologically diagnosed intravesical recurrence, that to diagnosed muscle invasive disease or upper tract urothelial carcinoma and that to death as a result of any cause, respectively.

Evaluation. All patients included in this study had cystoscopically confirmed tumors and/or positive findings on urinary cytology. In patients with intravesical lesions suggesting pure CIS alone, random bladder biopsy targeting the trigone, posterior wall, bilateral lateral walls, dome, anterior wall, prostatic urethra in men and/or suspicious regions, was performed, whereas the remaining patients with evidently visible tumors underwent complete TURBT. All pathological specimens were processed according to standard pathological procedures. Tumors were staged according to the 2002 American Joint Committee on Cancer TNM system and graded according to the 2004 World Health Organization/International Society of Urologic Pathology classification.

Treatment. As a rule, the second TUR was conducted for patients who were diagnosed with pT1 or high-grade tumor at our institution; however, omitting the second TUR was permitted after considering the clinical features of each patient such age and performance status. In addition, although the protocol for adjuvant intravesical instillation therapy was not strictly standardized, BCG therapy was generally performed for patients based on the physician's preference, patient tolerability and clinicopathological characteristics as previously reported (11). Follow-up of patients after TURBT of NMIBC was carried out as follows: Cystoscopy and urinary cytology were performed every 3-6 months for 2 years after TUR, and then every 6 months from 3 to 5 years. Upon detection of visible tumors or hyperemic mucosa by cystoscopy and/or positive findings on urinary cytology, transurethral biopsy of the abnormal region and/or TURBT of the tumor were performed.

Statistical analysis. All statistical analyses were performed using EZR software (Saitama Medical Center, Jichi Medical University, ver.1.36), and $\mathrm{P}<0.05$ were considered significant. The RFS, PFS and OS rates were calculated by the Kaplan-Meier method. The prognostic significance of certain factors was assessed by univariate and multivariate analyses using the Cox proportional hazards regression model. In this study, the factors that reached $\mathrm{P}<0.1$ in univariate analysis were included in multivariate analysis with backward stepwise selection.

\section{Results}

Patient characteristics. The characteristics of 186 patients included in this study are summarized in Table I. In this series, $47(25.3 \%)$ of the 186 patients underwent the second TUR following the initial TURBT, of whom 17 (36.2\%) were diagnosed as having residual urothelial carcinoma, including $1(2.1 \%)$ with muscle invasive disease. In addition, intravesical BCG therapy was performed for 108 patients $(58.1 \%)$, consisting of $96(88.9 \%)$ and $12(11.1 \%)$ receiving induction therapy alone and induction therapy followed by maintenance therapy, respectively.

Prognosis. During the observation period in this study (median, 39.6 months), disease recurrence, disease progression and overall deaths occurred in 54 (29.0\%), 14 (7.5\%) and $19(10.2 \%)$ patients, respectively. The RFS, PFS and OS rates in the 186 patients were as follows: 78.3,96.6 and 96.6\%, respectively, at 1 year, 68.9, 92.5 and $91.1 \%$, respectively, at 3 years, and 66.6, 90.2 and $87.2 \%$, respectively, at 5 years (Figs. 1, 2 and 3).

We then assessed the effects of several parameters, including age, sex, tumor multiplicity, tumor size, prior recurrent status, tumor grade, pathological $\mathrm{T}$ category, concomitant CIS, second TUR and intravesical BCG therapy, on the RFS, PFS and OS (Tables II, III and IV, respectively). Univariate analyses identified significant factors for oncological outcomes in the 186 patients as follows: BCG therapy for RFS $(\mathrm{P}=0.022)$, none for PFS, and age for OS $(\mathrm{P}=0.014)$. Furthermore, the following factors were demonstrated to be independently associated with the oncological outcomes in multivariate analyses: Tumor multiplicity and BCG therapy for RFS ( $\mathrm{P}=0.018$ and $\mathrm{P}=0.008$, respectively), tumor multiplicity and recurrence status for $\mathrm{PFS}(\mathrm{P}=0.043$ and $\mathrm{P}=0.029$, respectively), and age and tumor multiplicity for $\mathrm{OS}(\mathrm{P}=0.008$ and $\mathrm{P}=0.041$, respectively).

\section{Discussion}

Factors reflecting prognostic outcomes can be highly beneficial when counseling cancer patients to determine their therapeutic options and follow-up schedules. This is 
Table I. Patient characteristics.

\begin{tabular}{|c|c|}
\hline Variables & No of patients \\
\hline Mean age (range) (years) & $73(36-93)$ \\
\hline \multicolumn{2}{|l|}{ Sex } \\
\hline Male & 155 \\
\hline Female & 31 \\
\hline \multicolumn{2}{|l|}{ Number of tumors } \\
\hline Solitary & 82 \\
\hline Multiple & 104 \\
\hline \multicolumn{2}{|l|}{ Tumor diameter } \\
\hline$<3 \mathrm{~cm}$ & 152 \\
\hline$\geq 3 \mathrm{~cm}$ & 34 \\
\hline \multicolumn{2}{|l|}{ Recurrence status } \\
\hline Primary & 157 \\
\hline Recurrence & 29 \\
\hline \multicolumn{2}{|l|}{ Tumor grade } \\
\hline Low-grade & 19 \\
\hline High-grade & 167 \\
\hline \multicolumn{2}{|l|}{ T category } \\
\hline pTa & 76 \\
\hline pT1 & 91 \\
\hline pTis & 19 \\
\hline \multicolumn{2}{|l|}{ Concomitant CIS } \\
\hline Positive & 43 \\
\hline Negative & 143 \\
\hline \multicolumn{2}{|l|}{ Second TUR } \\
\hline Yes & 47 \\
\hline No & 139 \\
\hline \multicolumn{2}{|l|}{ BCG therapy } \\
\hline Yes & 108 \\
\hline No & 78 \\
\hline
\end{tabular}

CIS, carcinoma in situ; TUR, transurethral resection; BCG, bacillus of Calmette-Guerin.

especially true for patients with NMIBC, because they exhibit highly heterogeneous clinical courses with varying oncological outcomes, particularly those classified into the high-risk group (2-5). Although several major clinical guidelines clarified optimal therapeutic strategies for high-risk NMIBC patients, the oncological outcomes of these patients have not been well documented due to complicated therapeutic strategies (4). Accordingly, it is difficult to properly provide highly standardized treatments under strict criteria in clinical practice. In this study, therefore, we retrospectively analyzed the oncological outcomes of a total of 186 consecutive Japanese patients with high-risk NMIBC in order to investigate the impact of several clinicopathological parameters on these outcomes and to precisely clarify the therapeutic status of high-risk NMIBC.

To date, various factors predicting disease recurrence and progression have been reported for NMIBC to enable



Figure 1. Recurrence-free survival.

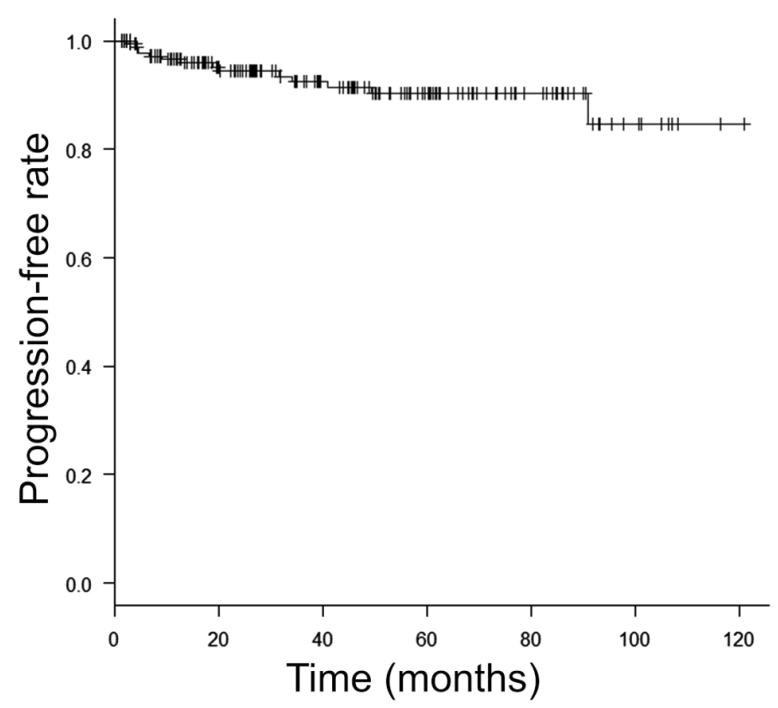

Figure 2. Progression-free survival.

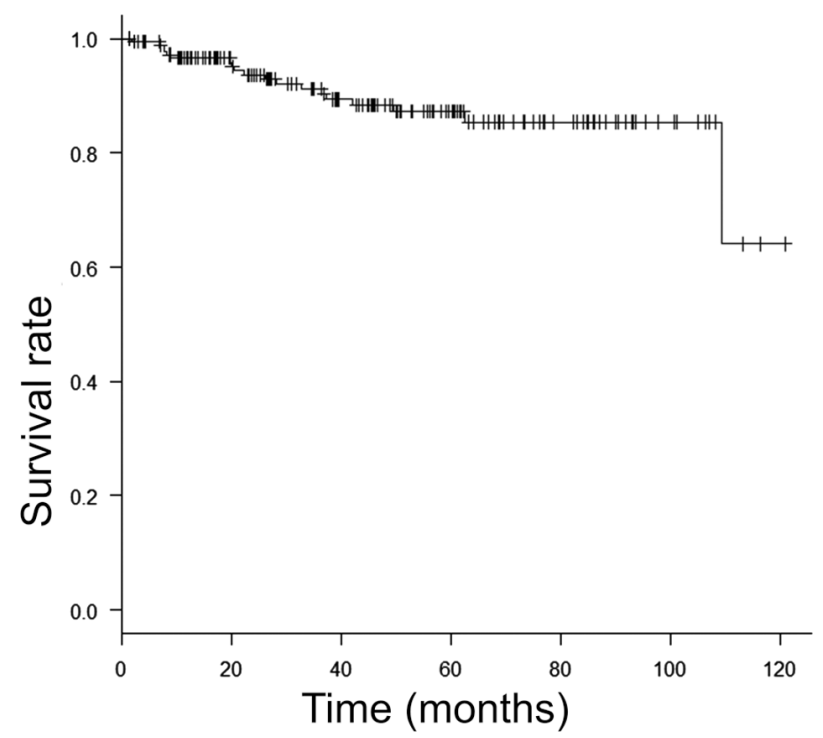

Figure 3. Overall survival. 
Table II. Prognostic factors for recurrence-free survival.

\begin{tabular}{|c|c|c|c|c|}
\hline \multirow[b]{2}{*}{ Variables } & \multicolumn{2}{|c|}{ Univariate analysis } & \multicolumn{2}{|c|}{ Multivariate analysis } \\
\hline & $\mathrm{HR}(95 \% \mathrm{CI})$ & P-value & $\mathrm{HR}(95 \% \mathrm{CI})$ & P-value \\
\hline Age, years (<70 vs. $\geq 70)$ & $1.33(0.76-2.35)$ & 0.31 & - & - \\
\hline Sex (male vs. female) & $1.43(0.64-3.16)$ & 0.38 & - & - \\
\hline Number of tumors (solitary vs. multiple) & $1.76(1.00-3.10)$ & 0.051 & $2.00(1.12-3.59)$ & 0.018 \\
\hline Tumor diameter (<3 vs. $\geq 3 \mathrm{~cm}$ ) & $1.80(0.99-3.26)$ & 0.053 & - & - \\
\hline Recurrence status (primary vs. recurrence) & $1.00(0.49-2.05)$ & 1.00 & - & - \\
\hline Tumor grade (low-grade vs. high-grade) & $0.59(0.27-1.31)$ & 0.20 & - & - \\
\hline T category (pTa, pTis vs. pT1) & $1.38(0.81-2.37)$ & 0.23 & - & - \\
\hline Concomitant CIS (yes vs. no) & $0.62(0.31-1.23)$ & 0.17 & - & - \\
\hline Second TUR (yes vs. no) & $0.79(0.41-1.50)$ & 0.46 & - & - \\
\hline BCG therapy (yes vs. no) & $0.54(0.31-0.92)$ & 0.022 & $0.48(0.28-0.82)$ & 0.008 \\
\hline
\end{tabular}

HR, hazards ratio; CI, confidence interval; CIS, carcinoma in situ; TUR, transurethral resection; BCG, bacillus of Calmette-Guerin.

Table III. Prognostic factors for progression-free survival.

\begin{tabular}{llccc}
\hline & \multicolumn{2}{c}{ Univariate analysis } & & Multivariate analysis \\
\cline { 2 - 3 } Variables & HR $(95 \%$ CI $)$ & P-value & HR (95\% CI) & P-value \\
\hline Age, years (<70 vs. $\geq 70)$ & $0.80(0.28-2.24)$ & 0.67 & - \\
Sex (male vs. female) & $0.97(0.22-4.37)$ & 0.97 & - \\
Number of tumors (solitary vs. multiple) & $3.19(0.89-11.5)$ & 0.076 & $3.78(1.04-13.8)$ \\
Tumor diameter (<3 vs. $\geq 3$ cm) & $0.29(0.038-2.22)$ & 0.23 & - \\
Recurrence status (primary vs. recurrence) & $0.28(0.92-8.41)$ & 0.071 & $3.46(1.14-10.5)$ \\
Tumor grade (low-grade vs. high-grade) & $1.21(0.16-9.35)$ & 0.85 & - \\
T category (pTa, pTis vs. pT1) & $2.63(0.82-8.41)$ & 0.10 & - \\
Concomitant CIS (yes vs. no) & $0.79(0.22-2.84)$ & 0.72 & - \\
Second TUR (yes vs. no) & $0.73(0.20-2.64)$ & 0.64 & - \\
BCG therapy (yes vs. no) & $1.03(0.34-3.08)$ & 0.96 & - \\
\hline
\end{tabular}

HR, hazards ratio; CI, confidence interval; CIS, carcinoma in situ; TUR, transurethral resection; BCG, bacillus of Calmette-Guerin .

satisfactory clinical decision-making based on an objective risk stratification system (6). Moreover, several models have been developed using multiple prognostic parameters to further individualize therapeutic strategies for NMIBC patients (6-8). Of these, the EORTC model integrated in the EAU guidelines may currently be the most commonly used (7). Thus, we used this system to identify 186 high-risk NMIBC patients treated at our institution. It was recommended to perform the second TUR followed by intensive adjuvant treatment, including intravesical BCG therapy and cystectomy, after initial TURBT for patients with high-risk NMIBC in the present major clinical guidelines $(4,9)$. However, only 25.3 and $58.1 \%$ of the 186 patients included in this study underwent the second TUR and BCG therapy, respectively, due to advanced age, unfavorable performance status, rejection of additional therapies and intolerance of BCG therapy. This suggested insufficient treatment for high-risk NMIBC patients at our institution; however, similar to this series, there have been several previous studies showing that a considerable proportion of NMIBC patients are not optimally managed according to current clinical guidelines (12-16). For example, a retrospective study including 23 centers in Western countries reported that the second TUR was carried out for only $38 \%$ of patients with T1G3 NMIBC (12), whereas SEER-Medicare data revealed that only $29 \%$ of patients with high-grade disease received BCG therapy (13).

The oncological outcomes in this series were comparatively favorable, with 5-year RFS, PFS and OS rates of $66.6,90.2$ and $87.2 \%$, respectively, and the proportion of patients with residual tumor during the second TUR was $36.2 \%$. These findings are comparable with those in previous studies $(8,12,17-21)$. For example, Kluth et al analyzed the prognostic outcomes in 892 patients with T1 high-grade NMIBC, and reported 5-year RFS, PFS and OS rates of 54,85 and $78 \%$, respectively (17). Similarly, Kikuchi et al reported 1-, 3- and 5-year RFS rates of 77.0, 
Table IV. Prognostic factors for overall survival.

\begin{tabular}{|c|c|c|c|c|}
\hline \multirow[b]{2}{*}{ Variables } & \multicolumn{2}{|c|}{ Univariate analysis } & \multicolumn{2}{|c|}{ Multivariate analysis } \\
\hline & $\mathrm{HR}(95 \% \mathrm{CI})$ & P-value & $\mathrm{HR}(95 \% \mathrm{CI})$ & P-value \\
\hline Age, years (<70 vs. $\geq 70)$ & $4.78(1.37-16.7)$ & 0.014 & $5.64(1.56-20.4)$ & 0.008 \\
\hline Sex (male vs. female) & $1.39(0.32-6.05)$ & 0.66 & - & - \\
\hline Number of tumors (solitary vs. multiple) & $2.46(0.88-6.83)$ & 0.085 & $2.96(1.05-8.40)$ & 0.041 \\
\hline Tumor diameter (<3 vs. $\geq 3 \mathrm{~cm}$ ) & $0.21(0.027-1.54)$ & 0.12 & - & - \\
\hline Recurrence status (primary vs. recurrence) & $1.32(0.44-3.99)$ & 0.62 & - & - \\
\hline Tumor grade (low-grade vs. high-grade) & $1.83(0.24-13.9)$ & 0.56 & - & - \\
\hline T category (pTa, pTis vs. pT1) & $1.48(0.59-3.71)$ & 0.40 & - & - \\
\hline Concomitant CIS (yes vs. no) & $1.29(0.49-3.42)$ & 0.61 & - & - \\
\hline Second TUR (yes vs. no) & $0.52(0.15-1.81)$ & 0.31 & - & - \\
\hline BCG therapy (yes vs. no) & $1.21(0.46-3.19)$ & 0.70 & - & - \\
\hline
\end{tabular}

HR, hazards ratio; CI, confidence interval; CIS, carcinoma in situ; TUR, transurethral resection; BCG, bacillus of Calmette-Guerin.

61.3 and $52.8 \%$, respectively, in 3237 Japanese patients with Ta or T1 bladder cancer (20). Furthermore, the proportion of patients diagnosed with residual tumor during the second TUR ranged between 33 and 44\% (20-22). Considering the low proportion of patients in this series treated in accordance with the current guidelines, the oncological outcomes were not inferior to those in previous report. Therefore, more satisfactory oncological outcomes may be expected for patients stratified into the high-risk group by appropriately providing the guideline-recommended standard treatments.

There have been several studies investigating prognostic factors in NMIBC patients with unfavorable characteristics (12,16-24). For example, Gontero et al identified age, tumor size and concomitant CIS as independent predictors of disease progression in T1G3 NMIBC patients initially treated by intravesical BCG instillation (12), whereas Branchereau et al identified lymphovascular invasion as a predictor of OS in patients with high-grade stage pT1 bladder cancer (24). To date, however, there is limited information regarding specific prognostic factors for high-risk NMIBC patients. Thus, it is of interest to explore useful parameters closely associated with oncological outcomes in high-risk NMIBC patients. In this series, multivariate analyses identified the following independent factors for poor oncological outcomes: Multiple tumors and absence of BCG therapy for RFS, multiple tumors and recurrent tumors for PFS, and elderly age and multiple tumors for OS. Considering the common effects of tumor multiplicity on the RFS, PFS and OS, high-risk NMIBC patients with multiple tumors should be treated more aggressively and followed more closely.

There were several limitations in this study. First, although the sample size was large for a cohort consisting of high-risk NMIBC patients alone, this was a retrospective study with a comparatively short observation period, and conducted in routine clinical practice without strict criteria regarding therapeutic strategy and follow-up schedule. Therefore, it will be of interest to perform further assessments by dividing patients with follow-up period $>5$ years according to whether treatments were performed based on current recommendations or not. Second, several recent studies demonstrated poor discrimination of disease recurrence and progression by currently accepted risk classification systems, including the EORTC risk tables, with an overestimation of these risks in high-risk patients in particular $(10,25)$. Thus, this point should be considered when interpreting the outcomes of this study. Lastly, as suggested by a number of previous studies (26-31), conventional clinicopathological parameters alone may be insufficient to precisely predict the oncological outcomes, suggesting the promising role of molecular biomarkers, such as Ki-67, cell cycle-associated proteins and neutrophil to lymphocyte ratio, for this objective.

In conclusion, we retrospectively investigated the oncological outcomes of a total of 186 Japanese patients with high-risk NMIBC. The 5-year RFS, PFS and OS were 66.6, 90.2 and $87.2 \%$, respectively, and the following factors were found to be independently associated with the oncological outcomes: Tumor multiplicity and introduction of BCG therapy for RFS, tumor multiplicity and recurrence status for PFS, and age and tumor multiplicity for OS. These findings suggest that favorable outcomes were achieved for the 186 patients; however, a more aggressive treatment schedule should be considered for patients with independent prognostic factors, especially those with multiple tumors.

\section{Acknowledgments}

Not applicable.

Funding

No funding was received.

\section{Availability of data and materials}

The datasets used and/or analyzed during the current study are available from the corresponding author on reasonable request. 


\section{Authors' contributions}

YM,HM and AO conceived the study and wrote the manuscript. TS and TI acquired and analyzed the general data of patients. $\mathrm{DM}$ and KT reviewed, collected data and assisted prognosis analysis. HM supervised the study, revised the manuscript and gave final approval of the version to be published. All authors have read and approved the final version of the manuscript..

\section{Ethics approval and consent to participate}

The current study was approved by the Ethics Committee of Hamamatsu University School of Medicine (Shizuoka, Japan; approval no. 14-290). Informed consent was waived due to the retrospective design of the present study. An opportunity for opt-out regarding the current study was provided through the institutional website.

\section{Patient consent for publication}

Not applicable.

\section{Competing interests}

The authors declare that they have no completing interests.

\section{References}

1. Kirkali Z, Chan T, Manoharan M, Algaba F, Busch C, Cheng L, Kiemeney L, Kriegmair M, Montironi R, Murphy WM, et al: Bladder cancer: Epidemiology, staging and grading, and diagnosis. Urology 66 (6 Suppl 1): S4-S34, 2005.

2. Audenet F, Attalla K and Sfakianos JP: The evolution of bladder cancer genomics: What have we learned and how can we use it? Urol Oncol 36: 313-320, 2018.

3. van den Bosch S and Alfred Witjes J: Long-term cancer-specific survival in patients with high-risk, non-muscle-invasive bladder cancer and tumour progression: A systematic review. Eur Urol 60: 493-500, 2011

4. Babjuk M,Böhle A, Burger M,Capoun O, Cohen D,Compérat EM, Hernández V, Kaasinen E, Palou J, Rouprêt M, et al: EAU guidelines on non-muscle-invasive urothelial carcinoma of the bladder: Update 2016. Eur Urol 71: 447-461, 2017.

5. Martin-Doyle W, Leow JJ, Orsola A, Chang SL and Bellmunt J: Improving selection criteria for early cystectomy in high-grade t1 bladder cancer: A meta-analysis of 15,215 patients. J Clin Oncol 33: 643-650, 2015.

6. Kluth LA, Black PC, Bochner BH, Catto J, Lerner SP, Stenzl A, Stenzl A, Sylvester R, Vickers AJ, Xylinas E and Shariat SF Prognostic and prediction tools in bladder cancer: A comprehensive review of the literature. Eur Urol 68: 238-253, 2015.

7. Sylvester RJ, van der Meijden AP, Oosterlinck W, Witjes JA, Bouffioux C, Denis L, Newling DW and Kurth K: Predicting recurrence and progression in individual patients with stage Ta T1 bladder cancer using EORTC risk tables: A combined analysis of 2596 patients from seven EORTC trials. Eur Urol 49: 466-477, 2006

8. Fernandez-Gomez J, Madero R, Solsona E, Unda M, Martinez-Piñeiro L, Gonzalez M, Portillo J, Ojea A, Pertusa C, Rodriguez-Molina J, et al: Predicting nonmuscle invasive bladder cancer recurrence and progression in patients treated with bacillus Calmette-Guerin: The CUETO scoring model. J Urol 182: 2195-2203, 2009.

9. Orsola A, Palou J and Solsona E: High-risk nonmuscle invasive bladder cancer. Hematol Oncol Clin North Am 29: 227-236, viii, 2015.

10. Xylinas E, Kent M, Kluth L, Pycha A, Comploj E, Svatek RS, Lotan Y, Trinh QD, Karakiewicz PI, Holmang S, et al: Accuracy of the EORTC risk tables and of the CUETO scoring model to predict outcomes in non-muscle-invasive urothelial carcinoma of the bladder. Br J Cancer 109: 1460-1466, 2013.
11. Hinotsu S, Akaza H, Naito S, Ozono S, Sumiyoshi Y, Noguchi S, Yamaguchi A, Nagamori S, Terai A, Nasu Y, et al: Maintenance therapy with bacillus Calmette-Guérin Connaught strain clearly prolongs recurrence-free survival following transurethral resection of bladder tumour for non-muscle-invasive bladder cancer. BJU Int 108: 187-195, 2011.

12. Gontero P, Sylvester R, Pisano F, Joniau S, Vander Eeckt K, Serretta V, Larré S, Di Stasi S, Van Rhijn B, Witjes AJ, et al: Prognostic factors and risk groups in T1G3 non-muscle-invasive bladder cancer patients initially treated with Bacillus Calmette-Guérin: Results of a retrospective multicenter study of 2451 patients. Eur Urol 67: 74-82, 2015.

13. Patschan O, Holmäng S, Hosseini A, Liedberg F, Ljungberg B, Malmström PU, Rosell J and Jahnson S: Use of bacillus Calmette-Guérin in stage T1 bladder cancer: Long-term observation of a population-based cohort. Scand J Urol 49: 127-132, 2015.

14. Spencer BA, McBride RB, Hershman DL, Buono D, Herr HW, Benson MC, Gupta-Mohile S and Neugut AI: Adjuvant intravesical bacillus Calmette-Guérin therapy and survival among elderly patients with non-muscle-invasive bladder cancer. J Oncol Pract 9: 92-98, 2013.

15. Witjes JA, Palou J, Soloway M, Lamm D, Kamat AM, Brausi M, Persad R, Buckley R, Colombel M and Böhle A: Current clinical practice gaps in the treatment of intermediate- and high-risk non-muscle-invasive bladder cancer (NMIBC) with emphasis on the use of bacillus Calmette-Guérin (BCG): Results of an international individual patient data survey (IPDS). BJU Int 112: 742-750, 2013.

16. Lenis AT, Donin NM, Litwin MS, Saigal CS, Lai J, Hanley JM, Konety BR and Chamie K; Urologic Diseases in America Project: Association between number of endoscopic resections and utilization of bacillus Calmette-Guérin therapy for patients with high-grade, non-muscle-invasive bladder cancer. Clin Genitourin Cancer 15: e25-e31, 2017.

17. Kluth LA, Xylinas E, Crivelli JJ, Passoni N, Comploj E, Pycha A, Chrystal J, Sun M, Karakiewicz PI, Gontero P, et al: Obesity is associated with worse outcomes in patients with T1 high grade urothelial carcinoma of the bladder. J Urol 190: 480-486, 2013.

18. Chamie K, Ballon-Landa E, Daskivich TJ, Bassett JC, Lai J, Hanley JM, Konety BR, Litwin MS and Saigal CS; Urologic Diseases in America Project: Treatment and survival in patients with recurrent high-risk non-muscle-invasive bladder cancer. Urol Oncol 33: 20.e9-20.e17, 2015.

19. Baltacı S, Bozlu M, Yıldırım A, Gökçe Mİ, Tinay İ, Aslan G, Can C, Türkeri L, Kuyumcuoğlu U and Mungan A: Significance of the interval between first and second transurethral resection on recurrence and progression rates in patients with high-risk non-muscle-invasive bladder cancer treated with maintenance intravesical bacillus Calmette-Guérin. BJU Int 116: 721-726, 2015.

20. Kikuchi E, Fujimoto H, Mizutani Y, Okajima E, Koga H, Hinotsu S, Shinohara N, Oya M and Miki T; Cancer Registration Committee of the Japanese Urological Association: Clinical outcome of tumor recurrence for Ta, T1 non-muscle invasive bladder cancer from the data on registered bladder cancer patients in Japan: 1999-2001 report from the Japanese urological association. Int J Urol 16: 279-286, 2009.

21. Grimm MO, Steinhoff C, Simon X, Spiegelhalder P, Ackermann R and Vogeli TA: Effect of routine repeat transurethral resection for superficial bladder cancer: A long-term observational study. J Urol 170: 433-437, 2003.

22. Jahnson S, Wiklund F, Duchek M, Mestad O, Rintala E, Hellsten S and Malmström PU: Results of second-look resection after primary resection of T1 tumour of the urinary bladder. Scand J Urol Nephrol 39: 206-201, 2005.

23. Lazica DA, Roth S, Brandt AS, Böttcher S, Mathers MJ and Ubrig B: Second transurethral resection after Ta high-grade bladder tumor: A 4.5-year period at a single university center. Urol Int 92: 131-135, 2014.

24. Branchereau J, Larue S, Vayleux B, Karam G, Bouchot O and Rigaud J: Prognostic value of the lymphovascular invasion in high-grade stage pT1 bladder cancer. Clin Genitourin Cancer 11: $182-188,2013$.

25. Hernández V, De La Peña E, Martin MD, Blázquez C, Diaz FJ and Llorente C: External validation and applicability of the EORTC risk tables for non-muscle-invasive bladder cancer. World J Urol 29: 409-414, 2011. 
26. Ding W, Gou Y, Sun C, Xia G, Wang H, Chen Z, Tan J, Xu K and Qiang D: Ki-67 is an independent indicator in non-muscle invasive bladder cancer (NMIBC); combination of EORTC risk scores and Ki-67 expression could improve the risk stratification of NMIBC. Urol Oncol 32: 42.e13-e19, 2014.

27. Liu B, Miyake H, Nishikawa M and Fujisawa M: Expression profile of epithelial-mesenchymal transition markers in non-muscle-invasive urothelial carcinoma of the bladder: Correlation with intravesical recurrence following transurethral resection. Urol Oncol 33: 110.e11-e18, 2015.

28. Behnsawy HM, Miyake H, Abdalla MA, Sayed MA, Ahmed Ael-F and Fujisawa M: Expression of cell cycle-associated proteins in non-muscle-invasive bladder cancer: Correlation with intravesical recurrence following transurethral resection. Urol Oncol 29: 495-501, 2011.

29. Yuk HD, Jeong CW, Kwak C, Kim HH and Ku JH: Elevated neutrophil to lymphocyte ratio predicts poor prognosis in non-muscle invasive bladder cancer patients: Initial intravesical bacillus calmette-guerin treatment after transurethral resection of bladder tumor setting. Front Oncol 8: 642, 2019.
30. Passoni N, Gayed B, Kapur P, Sagalowsky AI, Shariat SF and Lotan Y: Cell-cycle markers do not improve discrimination of EORTC and CUETO risk models in predicting recurrence and progression of non-muscle-invasive high-grade bladder cancer. Urol Oncol 34: 485.e7-485.e14, 2016.

31. Fahmy O, Khairul-Asri MG, Stenzl A and Gakis G: Systemic anti-CTLA-4 and intravesical Bacille-Calmette-Guerin therapy in non-muscle invasive bladder cancer: Is there a rationale of synergism? Med Hypotheses 92: 57-58, 2016. 